\title{
BMJ Open Bortezomib in antibody-mediated autoimmune diseases (TAVAB): study protocol for a unicentric, non- randomised, non-placebo controlled trial
}

\author{
Siegfried Kohler, ${ }^{1,2,3}$ Stefanie Märschenz, ${ }^{3}$ Ulrike Grittner, ${ }^{4}$ Tobias Alexander, ${ }^{5}$ \\ Falk Hiepe, ${ }^{5}$ Andreas Meisel ${ }^{1,2,3}$
}

To cite: Kohler S, Märschenz S, Grittner U, et al. Bortezomib in antibody-mediated autoimmune diseases (TAVAB): study protocol for a unicentric, nonrandomised, non-placebo controlled trial. BMJ Open 2019;9:e024523. doi:10.1136/ bmjopen-2018-024523

- Prepublication history for this paper is available online. To view these files, please visit the journal online (http://dx.doi. org/10.1136/bmjopen-2018024523).

Received 30 May 2018 Revised 27 November 2018 Accepted 7 December 2018

Check for updates

(c) Author(s) (or their employer(s)) 2019. Re-use permitted under CC BY-NC. No commercial re-use. See rights and permissions. Published by BMJ.

For numbered affiliations see end of article.

Correspondence to

Siegfried Kohler;

siegfried.kohler@charite.de

\section{ABSTRACT}

Introduction The clinical characteristics of autoantibodymediated autoimmune diseases are diverse. Yet, medical treatment and the associated complications are similar, that is, the occurrence of long-term side effects and the problem that a significant proportion of patients are non-responders. Therefore, new therapeutic options are needed. Bortezomib, a proteasome inhibitor, is effective in the treatment of multiple myeloma and data from experimental models and case reports suggest an effect in the treatment of autoantibody-mediated autoimmunity. In our study, we will determine the effect of bortezomib treatment on a shared surrogate parameter for clinical efficacy, namely change in autoantibody levels, which we chose as primary parameter.

Methods and analysis We designed a phase lla trial with altogether $n=18$ treatment-refractory patients suffering from myasthenia gravis, systemic lupus erythematosus and rheumatoid arthritis that will be treated with bortezomib add-on to pre-existing therapy. Primary endpoint is the change in autoantibody levels 6 months after therapy. Secondary endpoints include concomitant medication, disease-specific clinical scores and measures of quality of life and activities of daily living.

Ethics and dissemination Safety parameters include neurophysiological and clinical signs of peripheral neuropathy as well as potential central nervous system side effects determined by olfactory and neuropsychological testing. The study has been approved by the local ethical committee and first participants have already been enrolled. This proof of concept study will contribute to improve our understanding of plasma cellspecific treatment approaches by assessing its safety and efficacy in reducing serum levels of antibodies known to mediate autoimmune disorders. We plan to publish the final results of our study in a peer reviewed journal and to present our findings at international conferences. Trial registration number NCT02102594.

\section{INTRODUCTION}

In spite of a diversity of clinical manifestations, a number of autoimmune diseases are
Strengths and limitations of this study

- Our novel study design permits us to include three different autoantibody-mediated autoimmune diseases by using the change in autoantibody levels as shared primary endpoint

- This proof of concept study with small patient groups aims at generating data supporting a larger phase III trial.

- Safety measurements include a detailed neurophysiological and neuropsychological assessment in order to detect a possible affection of the peripheral or central nervous system.

characterised by a common pathophysiology with the production of pathogenic autoantibodies leading to an autoimmune attack on various body sites such as neuromuscular synapse, kidneys or joints. ${ }^{1-3}$ Treatment of autoantibody-mediated autoimmunity is similar across different medical fields and mainly relies on corticosteroids and additional immunosuppressive drugs like azathioprine, mycophenolate acid, cyclosporine and others. In recent years, the spectrum of available treatments has further been expanded by therapeutic antibodies targeting for example B cells or cytokines. ${ }^{4-6}$ However, current therapy often leads to unwanted side effects and still a significant fraction of patients do not respond adequately. Accordingly high-disease activity persists. This is probably due to the fact that especially so-called long-lived plasma cells are resistant to most current therapeutic options except autologous stem cell transplantation which is reserved for desperate cases. ${ }^{7}$ In spite of immunosuppressive therapy, these continuously antibody producing cells persist for years or even decades and are mainly 
responsible for disease chronicity and severity. Thus, there is a strong medical need for new therapeutic option alternatives concerning these diseases.

Bortezomib (Velcade) has been approved for the treatment of multiple myeloma (plasmocytoma). ${ }^{8}$ Its mechanism of action, the inhibition of the proteasome, leads to apoptosis in cells that have a high-protein turnover like for example tumour and myeloma cells. ${ }^{9}$ Importantly, plasma cells, having a high-protein turnover due to constant secretion of antibodies, are sensitive to bortezomib. In line with this, recent studies in experimental models show significant effects of bortezomib on autoantibody-mediated autoimmune diseases like systemic lupus erythematosus (SLE) or myasthenia gravis (MG). ${ }^{10-12}$ Furthermore, recent reports on case series' of bortezomib-treated SLE and NMDA enzephalitis patients support a beneficial role for bortezomib in the treatment of autoantibody-mediated autoimmunity. ${ }^{13}{ }^{14} \mathrm{~A}$ recent multicentre double-blind trial initiated to assess the effect of bortezomib in SLE could not report conclusive results with respect to efficacy, as discontinuation due to adverse events was extremely high. ${ }^{15}$ Yet, a prospective study investigating bortezomib in different forms of autoantibody-mediated autoimmunity in humans has never been conducted so far.

We postulate that bortezomib reduces plasma cell numbers and autoantibody production irrespective of specific antibody target or disease. Reduction of antibody levels should reduce disease activity in therapy-refractory patients suffering from antibody-mediated autoimmune diseases such as MG, SLE and anticitrullinated peptide/ protein antibodies (ACPA)-positive rheumatoid arthritis (RA).

These diseases have in common, that specific antibodies, that is, antiacetylcholine receptor (AChR), anti-doublestranded DNA (anti-ds-DNA) and ACPA, are not only diagnostic markers. They can also be used for measuring disease activity and therapeutic success, that is, clinical improvement that is intraindividually correlated with a reduction in respective autoantibody serum levels. ${ }^{16-23}$ Consequently, therapeutic measures for the treatment of acute disease relapses include plasmapheresis or immunadsorption aiming at a fast reduction of antibody levels.

\section{METHODS AND ANALYSIS Objective}

The goal of the TAVAB-trial is to examine the safety and therapeutic efficacy of bortezomib in patients with autoantibody-mediated autoimmune diseases refractory to currently available therapy. Our hypothesis is that bortezomib leads to a significant reduction in antibody titres, thus resulting in clinical improvement. Furthermore, a disease selective analysis of the effects of bortezomib will allow us to get knowledge of potential disease-specific effects. Depending on the results of this trial, a confirmatory phase III study can be envisioned.

\section{Study design}

The TAVAB trial is an investigator-initiated, prospective, unicentric, non-randomised, non-placebo controlled, one-armed proof-of-concept study. Altogether 18 patients, that is, $\mathrm{n}=6$ suffering from MG, SLE and RA refractory to current standard treatment strategies will be treated in an add-on manner with bortezomib. Bortezomib will be applied in two treatment cycles with four subcutaneous injections of $1.3 \mathrm{mg}$ bortezomib $/ \mathrm{m}^{2}$ body surface. In the case of hepatic insufficiency or peripheral neuropathy, the drug dose will be reduced according to the manufacturer's specifications. For prevention of herpes virus reactivation or Pneumocystis jerovici, during the bortezomib cycles and an additional period of 3 weeks, treatment of patients with $200 \mathrm{mg}$ of aciclovir daily and $960 \mathrm{mg}$ of trimethoprim/sulfamethoxazol three times a week is mandatory.

In between the two treatment cycles, there is a time interval of 10 days. The first follow-up visit is scheduled 10 days after the last bortezomib application, then monthly follow-up visits are scheduled for another 6 months. This is followed by two optional follow-up visits in 6-month intervals. The following parameters will be measured: vital signs, basic clinical status, concomitant medication, current disease-specific symptoms and scores, quality of life and activities of daily living scores, basic laboratory parameters, disease-specific antibody titres (anti-AChR, anti-ds-DNA, anti-ACPA), protective antibody serum levels (measles, tetanus, diphtheria and mumps), signs for an affection of the central or peripheral nervous system, adverse events and specific immunological parameters, eg, numbers of circulating plasma cells/blasts and the distribution of $\mathrm{B}$ cell, $\mathrm{CD} 4$ and $\mathrm{CD} 8 \mathrm{~T}$ cell and dendritic cell subsets (see figure 1).

\section{Patient and public involvement}

The study was designed based on the need for new therapeutic options for autoantibody-mediated autoimmune diseases in patients refractory to standard treatment. Patient-oriented secondary endpoints include questionnaires assessing disease-specific quality of life and activities of daily living. Patients were not actively involved in the study design, recruitment or conduct. After final analysis, study results will be explained to study participants on request.

There was no public involvement in the study design.

\section{Inclusion and exclusion criteria}

Inclusion criteria

For group I (MG)

- Confirmed diagnosis of MG, according to the guidelines of the Deutsche Gesellschaft für Neurologie.

- Only treatment-refractory patients with significant disease activity (eg, QMG of $\geq 10$ at screening) will be included, for example, at study entry a long-term immunosuppressive therapy excluding corticosteroids such as azathioprine or mycophenolat mofetil must have been used for at least 1 year. Alternatively, 
aciclovir ( $1 \mathrm{x} / \mathrm{d})$ and cotrimoxazol $(3 \mathrm{x} / \mathrm{w})$

\begin{tabular}{|c|c|c|c|c|c|c|c|c|c|c|c|c|c|c|c|c|}
\hline & & & & & & & & & & & & & & & \\
\hline & & \multicolumn{4}{|c|}{ 1. cycle bortezomib } & \multicolumn{4}{|c|}{ 2. cycle bortezomib } & & & & & & & \\
\hline & & B & B & B & B & B & B & $\mathrm{B}$ & B & & & & & & & \\
\hline & $\begin{array}{c}-d-0 \\
-d\end{array}$ & $\mathrm{~d} 1$ & d4 & d8 & $\mathrm{d} 11$ & $\mathrm{~d} 21$ & $\mathrm{~d} 24$ & d28 & d31 & $\begin{array}{c}1 \\
+10 d\end{array}$ & $\begin{array}{c} \\
+1 m\end{array}$ & $+1 m$ & $\begin{array}{c}1 \\
+1 m\end{array}$ & $+1 \mathrm{~m}$ & $+1 \mathrm{~m}$ & $\begin{array}{c}1 \\
+1 \mathrm{~m}\end{array}$ \\
\hline parameter & V1 & V2 & V3 & V4 & V5 & V6 & V7 & V8 & V9 & V10 & V11 & V12 & V13 & V14 & V15 & V16 \\
\hline disease score & $x$ & $x$ & & $x$ & & $x$ & & $x$ & & $x$ & $x$ & $x$ & $x$ & $x$ & $x$ & $x$ \\
\hline AdL, QoL & $x$ & $x$ & & & & $x$ & & & & & $x$ & & $x$ & & $x$ & $x$ \\
\hline antibodies & $x$ & $x$ & & & & $x$ & & & & $x$ & $x$ & $x$ & $x$ & $x$ & $x$ & $x$ \\
\hline PNP testing & $x$ & $x$ & & & & $x$ & & & & $x$ & & $x$ & & $x$ & & $x$ \\
\hline nerve conduction & & $x$ & & & & & & & & $x$ & & & & & & \\
\hline olfactory testing & & $x$ & & & & & & & & $x$ & & & & & & \\
\hline NP-testing & & $x$ & & & & & & & & $x$ & & & & & & \\
\hline
\end{tabular}

Figure 1 Intervention and visit schedule for the TAVAB trial. AdL, activities of daily living; NP-testing, neuropsychological testing; PNP testing, clinical examination for signs of polyneuropathy, that is, examination of tendon reflexes, examination of the sensory system and motor examination; QoL, quality of life.

if the patient does not take any immunosuppressive therapy at study entry, at least one type of immunosuppression must have been used in the past that had been stopped due to inefficacy or side effects.

- The patient must have been hospitalised at least once in the past for the administration of immunoglobulins or plasma exchange therapy due to myasthenic crisis.

- Detection of anti-AchR antibodies in serum at study entry.

- At the time point of administration of bortezomib, there must be a minimum interval of 1 month to the last administration of immunoglobulins or plasma exchange.

\section{For group II (SLE)}

- Confirmed diagnosis of SLE based on American College of Rheumatology (ACR) criteria.

- Detection of anti-dsDNA antibodies in serum.

- Classification into 'therapy refractory' is defined by elevated disease activity measured by an SLE Disease Activity Index (SLEDAI) score $\geq 8$ in spite of immunosuppression (intravenous cyclophosphamide, azathioprine or mycophenolat mofetil in standard dosage for more than 3 months).

- At the time point of administration of bortezomib, there must be a minimum interval of 3 months to the last administration of high-dose corticosteroid therapy or plasma exchange.

\section{For group III (RA)}

- Confirmed diagnosis of RA based on EULAR/ACR criteria.
- Classification into 'therapy refractory' is defined by a significant disease activity for at least 6 months with a Disease Activity Score 28 (DAS-28) $\geq 3.2$, in spite of at least two disease modifying anti rheumatic drugs in the past.

- Detection of Rheumatoid factor and/or ACPA antibodies in serum.

- At the time point of administration of bortezomib, there must be a minimum interval of 3 months to the last administration of high-dose corticosteroid therapy or plasma exchange.

For all groups

- Age from 18 to 75 years at screening.

- Able and willing to provide informed consent.

- If in reproductive age, patients and their respective partners must use a highly effective contraceptive method, alternatively an abstinence of heterosexual activity must be confirmed.

- Negative urine pregnancy test at screening (if applicable).

\section{Exclusion criteria}

For group I (MG)

History of thymectomy less than 1 year before study entry.

\section{For group III (RA)}

- Application of intra-articular or systemic corticosteroids for the treatment of an acute exacerbation within the last 4 weeks before screening if not administered in the frame of a regular therapeutic regimen. 
- Tumour necrosis factor-alpha blockade within the last 3 months before screening.

- Tocilizumab therapy within the last 3 months before screening.

- Abatacept therapy within the last 3 months before screening.

\section{For groups I-III}

- B-cell-depletion therapy in the past 9 months.

- Renal insufficiency with a glomerular filtration rate lower than $40 \mathrm{~mL} / \mathrm{min}$.

- Congestive heart failure with a confirmed left ventricular ejection fraction $<40 \%$.

- Uncontrolled cardiac arrhythmia.

- Elevated pulmonal arterial pressure $>50 \mathrm{~mm} \mathrm{Hg}$, TLCO/VA $<40 \%$.

- Liver cirrhosis (child A, B or C).

- Pre-existing sensory or motoric polyneuropathy $\geq$ grade 2 according to NCI CTC AE criteria within 14 days before study entry.

- Signs of a herpes zoster reactivation at study entry or history of severe reactivation, that is, involving eye, ear, brain or a large body surface.

- Persons with either a currently active systemic infection or with history of a severe viral infection (CMV, $\mathrm{EBV}$ ) in the past 6 months before screening.

- Active hepatitis B and/or C or known HIV infection.

- Current or past (up to 5 years before study entry) malignancy, except multiple myeloma, thymoma/ thymic-carcinoma.

- Known bortezomib, trimethoprim/sulfamethoxazol, aciclovir allergy or intolerance.

- Patients with a clinically significant liver, kidney or bone marrow dysfunction defined by the following laboratory values at screening.

- Haemoglobin $<8.5 \mathrm{~g} / \mathrm{L}$.

- White cells count $<2.5 \times 10^{9} / \mathrm{L}$.

- Thrombocytes $<100 / \mathrm{nL}$.

- ASAT/ALAT >3.5x higher than upper reference values, except in the case of an SLE relapse or as side effect of ongoing medication with for example, azathioprin.

- Bilirubin $>2.0 \mathrm{mg} / \mathrm{dL}$.

- Pregnancy or breast feeding.

- Presence of any comorbidity, which in the opinion of the principal investigator could significantly decrease the ability of the patient to tolerate the planned therapy.

- Any medical, psychiatric or other condition, that significantly limits the patient's capability to understand the patient information, to declare informed consent, to comply with the study protocol or to complete the study.

- Patients who are imprisoned or institutionalised.

- Missing permission to save and process pseudonymised data in the study.

- Current or past (last 3 months) participation in another interventional study.

\section{Primary and secondary endpoints}

The primary endpoint is defined as the change in serum concentration of the respective pathogenic antibody in each of the examined diseases (ie, anti-AChR in MG, antidsDNA in SLE and anti-ACPA in RA) after bortezomib treatment. More precisely, the intraindividual relative change in serum titres comparing measurement before first application of bortezomib to values 6 month after the treatment (V16) is calculated. All antibody titres are measured by ELISA in a clinical laboratory with official accreditation for these tests.

Secondary endpoints include the change in specific autoantibody titres measured before treatment compared with multiple time points in the course of the study. Clinical parameters are based on a regular physical examination as well as disease-specific clinical scores. In detail, these are the MGFA classification at the time of presentation, QMG Score, SLEDAI Score, BILAG Score, SLE Responder Index, RA DAS-28 and ACR Response Criteria. Furthermore, secondary endpoints include disease-specific assessment of Quality of Life and Activities of Daily Living by MG-Qol, MG-ADL, Lupus-Qol, RA-Qol, HAQ (Health assessment questionnaire) for SLE and RA. Additionally, comedication is analysed and treatment effects on global humoral immunity are measured by analysis of protective antibody titres.

Furthermore, a detailed monitoring of changes of a variety of immunological parameters, for example, number of activated plasma cells, distribution of B cell, CD4 and CD8 T cell and dendritic cell subsets and concentrations of soluble mediators is included in the study.

\section{Statistical methods}

The trial is designed as proof of concept study and does not aim at detecting a statistically significant clinical efficacy with respect to disease-specific scores. However, the primary hypothesis considers antibody serum levels as surrogate parameter for changes in intraindividual disease activity, which is expected to be significantly reduced by bortezomib. A further aim is to gather data in order to enable a power analysis and sample size planning for a later phase III trial proving the capacity of bortezomib in improving clinical outcome.

Relative change of the disease-specific antibody serum levels compared 6 months before and after completion of bortezomib treatment was defined as primary endpoint. As for all the diseases in the focus of this study, antibody levels are considered as surrogate parameter for clinical activity, for each individual patient, the relative change is calculated and the statistical analysis of the primary endpoint does not discriminate between the three diseases.

Altogether, 18 patients will be enrolled in the study. With respect to data from a case series of SLE patients treated with bortezomib, we expect a mean reduction of pathogenic antibody titre of around 60\% (SD $20 \%)$. This equals an effect size of 3 . With this assumption and an expected drop-out rate of around 20\%, a 
significant reduction should be detectable (two-sided testing, alpha $=0.05$, paired $t$ test and power $99 \%$ ).

Secondary endpoints are expected changes in the following parameters:

- (a reduction in) disease severity (clinical score).

- (an increase in) quality of life (QoL-score).

- (an increase in) abilities concerning activities of daily living (AdL-score).

- (a reduction in) dosage of immunosuppressive comedication.

- (a reduction in) protective antibodies (mumps, measles, rubella, VZV, pneumococci, CMV).

- (a reduction in) autoantibody titres at all single time points during and after completion of bortezomib administration as compared with baseline.

- (a reduction in) numbers of antibody producing plasmablasts/cells and concentration of soluble mediators in peripheral blood.

The aforementioned secondary endpoints are measured at multiple time points after bortezomib application and changes are determined in comparison to before start of treatment. All parameters are analysed for the specific diseases in an explorative fashion.

The descriptive analysis of secondary endpoints for the single time points is conducted by the determination of mean values and SD in the case of normally distributed data or alternatively by median and interquartile ranges.

\section{Safety}

Safety monitoring includes vital signs, clinical examination for signs of polyneuropathy, that is, examination of tendon reflexes, examination of the sensory system and motor examination, ECG, routine laboratory analysis and registration of adverse events. Neurophysiological measurement of nerve conduction, assessment/evaluation of olfactory function and neuropsychological testing before and after completed bortezomib administration specifically aims at detecting an affection of the peripheral or central nervous system.

\section{Dissemination}

The study protocol is published in this journal. Independent of study outcome the final results will be made available to the public in a peer-reviewed, international journal.

\section{DISCUSSION}

Current therapeutic options for autoantibody-mediated autoimmunity are not able to directly attack the main culprit for disease pathogenesis and chronicity, namely the autoantibody producing plasma cell. ${ }^{7}$ Yet, based on its mechanism of action, ${ }^{9}$ experimental evidence ${ }^{10-12}$ and case reports, ${ }^{13} 14$ we believe that bortezomib might offer an interesting new therapeutic option. Thus, we have initiated the TAVAB trial, aiming to investigate the therapeutic potential of inhibition of the proteasome by bortezomib in severely affected MG, SLE and RA patients refractory to standard therapy. One special aspect of this study is that we are testing a new treatment alternative in three different indications using a primary endpoint relevant to all three diseases. This is only possible, as the three diseases have in common that antibody levels can be regarded as surrogate parameter for clinical disease severity. ${ }^{16-26}$ By determining relative changes of autoantibody serum levels before and after bortezomib therapy, we can calculate data from all included patients to prove the efficacy of our drug with respect to the primary endpoint of our study regardless of the individual disorder.

In contrast to our first study in $\mathrm{SLE}^{1315}$ and as sole alteration to the usual protocol for the treatment of MM with bortezomib, we do not co-administer dexamethasone to our patients. The reason for this is that we want to rule out that changes in serum antibody titre are caused rather by dexamethasone than by bortezomib itself. In general, we use a protocol comparable to the study by Ishii $e a^{15}$ that has recently reported a high drop-out rate due to side effects. It remains to be seen whether our study will be affected by a similar drop-out rate and whether this could be an SLE-specific problem.

In the meantime, the study has already started, enrolment for SLE and MG patients is as expected and the envisioned enrolment numbers for these two indications should be fulfilled in 2018. However, so far we are unable to include any RA patient in our study, even after changing our inclusion criteria for RA patients. This might be due to the fact, that new treatment options, that is, JAK inhibitors, have been approved for the treatment of RA.

In order to overcome this problem, we have changed our study protocol in accordance with the responsible regulatory institutions. We have decided to keep the total number of 18 patients constant; however, we will now include patients with either MG, SLE or RA on a firstcome first-serve basis. This will result in more patients with MG and SLE, while the number of included RA patients will be lower. In the most extreme scenario, no RA patient will be included and therefore RA disease-specific effects of bortezomib will be difficult to estimate. Yet, by keeping the total number of included patients constant, we will still be able to analyse safety and therapeutic efficacy of bortezomib in patients with autoantibody-mediated autoimmune diseases in general. As the number of bortezomib-treated MG and SLE patients in our new study protocol is increased, we have the possibility to reveal specific effects for these diseases on an even stronger basis. Certainly, the small number of included patients might still be limiting.

Bortezomib has already been used for treatment of multiple myeloma for several years; therefore, potential side effects are quite well known. Consequently, the treating physicians and affected patients have the possibility to estimate the potential benefit of treatment relative to the risk of potential side effects. Due to the current experiences with this substance, we assume that especially a peripheral neuropathy and a reactivation of herpes virus might be a potential risk for our patients. However, 
bortezomib induced neuropathy is often reversible ${ }^{27-29}$ and in less severe cases even a dose adjustment is sufficient for the prevention of long-lasting nerve damage. Reactivation of herpes virus should be preventable by prophylactic treatment with aciclovir incorporated in our study design.

In our study, we have included two tests in order to specifically monitor effects of bortezomib on the central nervous system. For this purpose, we will evaluate olfactory and neuropsychological performance before and after bortezomib administration. All in all, during the whole study, the patients are carefully monitored for side effects and therefore we believe that the risk of serious long-lasting side effects is acceptably low.

In conclusion, we consider our study to provide a good chance for paving the way for novel therapeutic strategies in autoantibody-mediated disorders. Patients to be included in our study are afflicted with a severe course of chronic disorder often needing frequent hospitalisations and impairing patients' quality of life dramatically. These patients are usually treated on a long term with the current standard therapy sometimes accompanied by (severe) side effects. We do not aim at proving a significant clinical improvement in this study. Yet, we consider our primary endpoint, which is a significant intraindividual reduction of autoantibody serum levels through bortozomib application, important to judge whether bortezomib has the capacity to improve the clinical course in autoantibody-mediated disorders. Consequently, our study will hopefully enable us to justify and plan a phase III clinical trial to prove the efficacy of bortezomib in the treatment of MG, RA and SLE.

\section{Trial status}

The current protocol (V.1.4) was approved on 24 August 2017. The first protocol version had been approved on 17 July 2014. Study recruitment began on 31 October 2014 and is expected to be completed by June 2019 .

\section{Author affiliations}

${ }^{1}$ Department of Experimental Neurology, Charité - Universitätsmedizin Berlin, Berlin, Germany

${ }^{2}$ Department of Neurology, Charité - Universitätsmedizin Berlin, Berlin, Germany ${ }^{3}$ Neurocure Clincal Research Center, Charite Universitätsmedizin Berlin, Berlin, Germany

${ }^{4}$ Institute of Biometry and Clinical Epidemiology, Charité - Universitätsmedizin Berlin, Berlin, Germany

${ }^{5}$ Department of Rheumatology, Charité - Universitätsmedizin Berlin, Berlin, Germany

Acknowledgements The authors would like to thank all study participants.

Contributors SK, TA, FH and AM conceptualised the study. SK, SM, UG, TA, FH and AM wrote the study protocol, UG was responsible for statistical calculations. All authors (SK, SM, UG, TA, FH and AM) wrote, edited and approved the manuscript.

Funding This work was supported by the Deutsche Forschungsgemeinschaft (DFG) through grants EXC 257 (AM) and the SFB650 TP17 (FH, AT) und TRR130 TP15 (FH, AT). We acknowledge support from the German Research Foundation (DFG) and the Open Access Publication Fund of Charité - Universitätsmedizin Berlin for publication costs. The funding source has no influence on trial design, trial conduct, data handling, data analysis or writing of the manuscript. The authors will have access to the final database of the trial.
Competing interests AM received honoraria for serving on the scientific advisory board of Alexion, and he served on the speaker's bureau of Novartis, Alexion and Grifols. All other authors report no conflict of interest related to this study.

Patient consent for publication Not required.

Ethics approval The study protocol was reviewed and approved by the local ethical committee, that is, the Ethik-Kommission des Landes Berlin, and by the Bundesinstitut für Arzneimittel und Medizinprodukte (BfArM).

Provenance and peer review Not commissioned; externally peer reviewed.

Open access This is an open access article distributed in accordance with the Creative Commons Attribution Non Commercial (CC BY-NC 4.0) license, which permits others to distribute, remix, adapt, build upon this work non-commercially, and license their derivative works on different terms, provided the original work is properly cited, appropriate credit is given, any changes made indicated, and the use is non-commercial. See: http://creativecommons.org/licenses/by-nc/4.0/.

\section{REFERENCES}

1. Querol L, Illa I. Myasthenia gravis and the neuromuscular junction. Curr Opin Neurol 2013;26:459-65.

2. Tsokos GC, Lo MS, Costa Reis P, et al. New insights into the immunopathogenesis of systemic lupus erythematosus. Nat Rev Rheumatol 2016;12:716-30.

3. Derksen V, Huizinga TWJ, van der Woude D. The role of autoantibodies in the pathophysiology of rheumatoid arthritis. Semin Immunopathol 2017;39:437-46.

4. Hehir MK, Hobson-Webb LD, Benatar M, et al. Rituximab as treatment for anti-MuSK myasthenia gravis: Multicenter blinded prospective review. Neurology 2017;89:1069-77.

5. Leone A, Sciascia S, Kamal A, et al. Biologicals for the treatment of systemic lupus erythematosus: current status and emerging therapies. Expert Rev Clin Immunol 2015;11:109-16.

6. Tandan R, Hehir MK, Waheed W, et al. Rituximab treatment of myasthenia gravis: A systematic review. Muscle Nerve 2017;56:185-96.

7. Hiepe F, Dörner T, Hauser AE, et al. Long-lived autoreactive plasma cells drive persistent autoimmune inflammation. Nat Rev Rheumatol 2011;7:170-8.

8. Richardson PG, Sonneveld P, Schuster MW, et al. Bortezomib or high-dose dexamethasone for relapsed multiple myeloma. $N$ Engl $J$ Med 2005;352:2487-98.

9. Goy A, Gilles F. Update on the proteasome inhibitor bortezomib in hematologic malignancies. Clin Lymphoma 2004;4:230-7.

10. Neubert K, Meister S, Moser K, et al. The proteasome inhibitor bortezomib depletes plasma cells and protects mice with lupus-like disease from nephritis. Nat Med 2008;14:748-55.

11. Gomez AM, Vrolix K, Martínez-Martínez P, et al. Proteasome inhibition with bortezomib depletes plasma cells and autoantibodies in experimental autoimmune myasthenia gravis. J Immunol 2011;186:2503-13.

12. Gomez AM, Willcox N, Vrolix K, et al. Proteasome inhibition with bortezomib depletes plasma cells and specific autoantibody production in primary thymic cell cultures from early-onset myasthenia gravis patients. J Immunol 2014;193:1055-63.

13. Alexander T, Sarfert R, Klotsche J, et al. The proteasome inhibitior bortezomib depletes plasma cells and ameliorates clinical manifestations of refractory systemic lupus erythematosus. Ann Rheum Dis 2015;74:1474-8.

14. Scheibe F, Prüss H, Mengel AM, et al. Bortezomib for treatment of therapy-refractory anti-NMDA receptor encephalitis. Neurology 2017;88:366-70.

15. Ishii T, Tanaka Y, Kawakami A, et al. Multicenter double-blind randomized controlled trial to evaluate the effectiveness and safety of bortezomib as a treatment for refractory systemic lupus erythematosus. Mod Rheumatol 2018;28:986-92.

16. Lefvert AK, Bergström K, Matell G, et al. Determination of acetylcholine receptor antibody in myasthenia gravis: clinical usefulness and pathogenetic implications. J Neurol Neurosurg Psychiatry 1978;41:394-403.

17. Tindall RS. Humoral immunity in myasthenia gravis: effect of steroids and thymectomy. Neurology 1980;30:557-7.

18. Seybold ME, Lindstrom JM. Patterns of acetylcholine receptor antibody fluctuation in myasthenia gravis. Ann N Y Acad Sci 1981;377:292-306.

19. Vincent A, Newsom-Davis J, Newton P, et al. Acetylcholine receptor antibody and clinical response to thymectomy in myasthenia gravis. Neurology 1983;33:1276-82. 
20. ter Borg EJ, Horst G, Hummel EJ, et al. Measurement of increases in anti-double-stranded DNA antibody levels as a predictor of disease exacerbation in systemic lupus erythematosus. A long-term, prospective study. Arthritis Rheum 1990;33:634-43.

21. Besinger UA, Toyka KV, Hömberg M, et al. Myasthenia gravis: longterm correlation of binding and bungarotoxin blocking antibodies against acetylcholine receptors with changes in disease severity. Neurology 1983;33:1316-21.

22. Oosterhuis HJ, Limburg PC, Hummel-Tappel E, et al. Antiacetylcholine receptor antibodies in myasthenia gravis. Part 2. Clinical and serological follow-up of individual patients. J Neurol Sci 1983;58:371-85.

23. Oosterhuis HJ, Limburg PC, Hummel-Tappel E, et al. Antiacetylcholine receptor antibodies in myasthenia gravis. Part 3. The effect of thymectomy. J Neurol Sci 1985;69:335-43.

24. Chen HA, Lin KC, Chen $\mathrm{CH}$, et al. The effect of etanercept on anti-cyclic citrullinated peptide antibodies and rheumatoid factor in patients with rheumatoid arthritis. Ann Rheum Dis 2006;65:35-9.
25. Alessandri C, Bombardieri M, Papa N, et al. Decrease of anti-cyclic citrullinated peptide antibodies and rheumatoid factor following antiTNFalpha therapy (infliximab) in rheumatoid arthritis is associated with clinical improvement. Ann Rheum Dis 2004;63:1218-21.

26. Kastbom A, Strandberg G, Lindroos A, et al. Anti-CCP antibody test predicts the disease course during 3 years in early rheumatoid arthritis (the Swedish TIRA project). Ann Rheum Dis 2004;63:1085-9.

27. Dimopoulos MA, Mateos MV, Richardson PG, et al. Risk factors for, and reversibility of, peripheral neuropathy associated with bortezomib-melphalan-prednisone in newly diagnosed patients with multiple myeloma: subanalysis of the phase 3 VISTA study. Eur $J$ Haematol 2011;86:23-31.

28. Morawska M, Grzasko N, Kostyra M, et al. Therapy-related peripheral neuropathy in multiple myeloma patients. Hematol Oncol 2015;33:113-9.

29. Tacchetti P, Terragna C, Galli M, et al. Bortezomib- and thalidomideinduced peripheral neuropathy in multiple myeloma: clinical and molecular analyses of a phase 3 study. Am J Hematol 2014;89:1085-91. 